\title{
Cumplimiento de la quimioprofilaxis antipalúdica en ASPFOR XXXIII
}

\author{
Arcos Sánchez C. ${ }^{1}$, Salinas Vela FT. ${ }^{2}$
}

Sanid. mil. 2015; 71 (2): 103-108; ISSN: 1887-8571

\begin{abstract}
RESUMEN
El Paludismo o Malaria en Afganistán es una enfermedad endémica por debajo de los 2.000 metros de altitud. En pleno siglo XXI la malaria continúa siendo una amenaza para los militares desplegados en Zona de Operaciones y puesto que la quimioprofilaxis adecuada con fármacos junto con los métodos para evitar la picadura del mosquito son las medidas que más protegen, es necesario hacer un esfuerzo por un mayor y mejor cumplimiento de las directrices en cuanto a quimioprofilaxis.
\end{abstract}

PALABRAS CLAVE: Paludismo, Malaria, Quimioprofilaxis.

Compliance with malaria chemoprophylaxis in ASPFOR XXXIII.

SUMMARY: Malaria in Afghanistan is an endemic disease in all areas lower than 2000 meters. In the XXI century it remains a threat to troops in combat zone despite the availability of effective drug prevention and keep out biting insects measures, as the most effective ones, it is necessary a higher effort to get a bigger and better observance of the chemoprophylaxis.

KEYWORDS: Malaria, Chemoprophylaxis.

\section{INTRODUCCIÓN}

El paludismo o malaria es una enfermedad producida por protozoos del género Plasmodium sp. (falciparum, ovale, vivax y malariae) y trasmitida por la picadura de la hembra del mosquito Anopheles infectada. El cuadro clínico que produce es muy variable, cursando con fiebre, escalofríos, nauseas y sudoración profusa. La malaria sigue siendo la enfermedad infecciosa que produce mayor morbilidad y mortalidad en el mundo.

Los vectores representan un papel esencial en la transmisión de muchas enfermedades infecciosas (los mosquitos y las moscas hematófagas son importantes insectos vectores de enfermedades, las garrapatas y ciertos caracoles acuáticos participan en el ciclo vital y de transmisión de enfermedades). En la Tabla 1 se exponen los principales vectores y las enfermedades que transmiten.

El paludismo supone un problema de salud pública en más de 90 países, implicando un riesgo para aproximadamente 2400 millones de personas ${ }^{1}$. Existen países considerados áreas endémicas, estimándose que el $36 \%$ de la población mundial vive en áreas de riesgo palúdico que afecta principalmente a países de África tropical, Sudeste Asiático, Oceanía, Haití, Amazonas, América Central, Oriente Medio, India y África Subsahariana, con riesgo heterogéneo variando de país a país e incluso en distintas zonas dentro del mismo país (Figura 1). Además se han

${ }^{1}$ Cte. Médico. Servicio de Neurología.

${ }^{2}$ Cte. Médico. Servicio de Radiodiagnóstico.

Hospital General de la Defensa "Orad y Gajías”. Zaragoza. España.

Dirección para correspondencia: Carolina Arcos Sánchez. Servicio de Neurología. Hospital General de la Defensa “Orad y Gajías” de Zaragoza. Vía Ibérica, 1, 50009 Zaragoza. España.carcsan@fn.mde.es

Recibido: 25 de febrero de 2014

Aceptado: 23 de septiembre de 2014 descrito casos de Paludismo en zonas no endémicas cercanas a aeropuertos, ya que, con los vuelos intercontinentales el mosquito puede viajar y transmitir la infección. Es una enfermedad en auge con un incremento de casos importados (30.000/año en países industrializados). En España se declaran alrededor de 300-400 casos/año, de los que el 45\% son inmigrantes. Más del $75 \%$ de todos los casos importados se deben a P. falciparum, la especie más virulenta.

El Ministerio de Sanidad, Servicios Sociales e Igualdad emite las recomendaciones en Sanidad Exterior en cuanto a quimioprofilaxis indicadas en los viajes según las de la Organización Mundial de la Salud (OMS) ${ }^{2,3}$. Ya que se trata de una enfermedad para la que no hay una vacuna disponible, lo fundamental es evitar la picadura del vector, lo que se lleva a cabo a través de la prevención primaria y la prevención secundaria ${ }^{4}$.

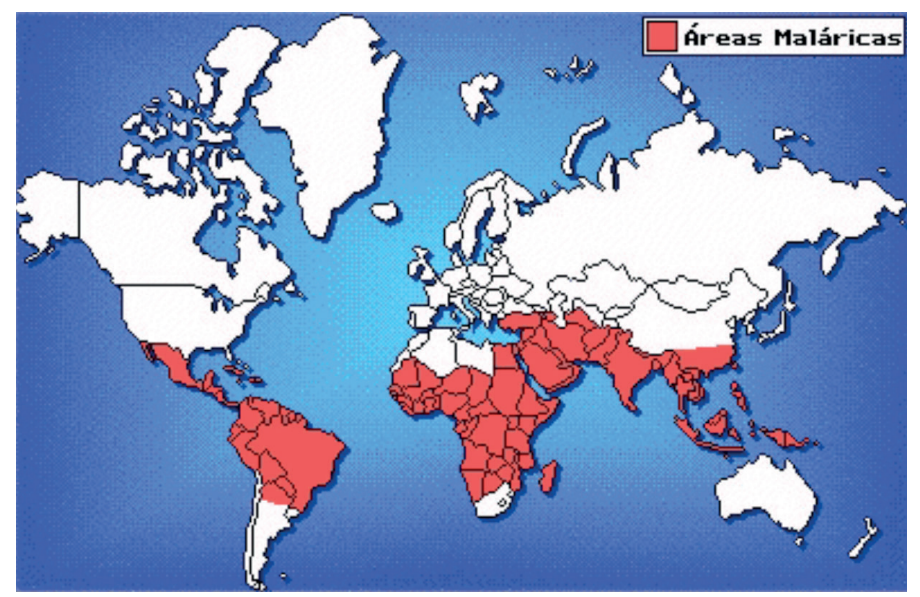

Figura 1. Áreas endémicas. 
Tabla 1. Principales vectores y enfermedades que transmiten ${ }^{11}$.

\begin{tabular}{|c|c|}
\hline Vectores & Principales enfermedades transmitidas(*) \\
\hline Caracol acuático & Esquistosomiasis (Bilharziasis) \\
\hline Mosca negra & Ceguera de río (Oncocercosis) \\
\hline Pulga & $\begin{array}{l}\text { Peste (transmitida por pulgas de ratas a humanos) } \\
\text { Rickettsiosis }\end{array}$ \\
\hline Mosquitos-Aedes & $\begin{array}{l}\text { Fiebre del Dengue } \\
\text { Fiebre del Valle del Rift } \\
\text { Fiebre amarilla } \\
\text { Chikungunya }\end{array}$ \\
\hline M-Anopheles & $\begin{array}{l}\text { Filariasis Linfática } \\
\text { Paludismo }\end{array}$ \\
\hline M- Culex & $\begin{array}{l}\text { Encefalitis Japonesa } \\
\text { Filariasis Linfática } \\
\text { Fiebre del Nilo Occidental }\end{array}$ \\
\hline Flebotomos hembras & $\begin{array}{l}\text { Leishmaniasis } \\
\text { Fiebre por flebótomos }\end{array}$ \\
\hline Garrapatas & $\begin{array}{l}\text { Fiebre hemorrágica Crimea- Congo } \\
\text { Enfermedad de Lyme } \\
\text { Fiebre recurrente (Borreliosis) } \\
\text { Enfermedades rickettsiales incluidas fiebre botonosa y fiebre Q } \\
\text { Encefalitis por Garrapatas } \\
\text { Tularemia }\end{array}$ \\
\hline Chinche triatoma & Enfermedad de Chagas (Tripanosomiasis Americana) \\
\hline Mosca Tsé- Tsé & Enfermedad del sueño (Tripanosomiasis Africana) \\
\hline
\end{tabular}

* Basado en extensas investigaciones, no existe absolutamente ninguna evidencia científica de que la infección por VIH pueda ser transmitida por insectos.
Como ya se ha mencionado los diferentes medicamentos y las pautas de administración se basarán en las recomendaciones de la Organización Mundial de la Salud6. La OMS distingue zonas con $P$. falciparum resistente a cloroquina y zonas con $P$. falciparum sensible a cloroquina. Existen otras resistencias a fármacos y de otros Plasmodium, siendo útil para conocerlas consultar la página web de la $\mathrm{OMS}^{7}$.

La prevención recomendada para cada país se decide basándose en los siguientes factores: el riesgo de contraer paludismo; las especies de parásitos del paludismo prevalentes en la zona; el nivel y extensión de las resistencias a medicamentos registradas en cada país; y el posible riesgo de efectos secundarios graves como consecuencia del uso de los diferentes medicamentos profilácticos ${ }^{8}$. Allí donde existe tanto $P$. falciparum como $P$. vivax es prioritaria la prevención de la malaria por falciparum. A menos que el riesgo de malaria se defina como "exclusivamente" debido a ciertas especies ( $P$. falciparum o $P$. vivax), los viajeros podrán infectarse de cualquier parásito, incluyendo las infecciones mixtas.

Además hay que tener en cuenta que a pesar de tomar cualquier medicación preventiva, la posibilidad de contraer paludismo no desaparece

\section{Prevención primaria}

- Medidas de Barrera: El estímulo principal de atracción vectorial es olfatorio, también térmico y visual (secreción de $\mathrm{CO}$, ácido láctico, perfumes, jabones y cremas). Vestir mangas largas, pantalones largos, evitar ropas de colores oscuros.

- Repelentes de Insectos: 35\%-40\% DEET (3-4h); IcaridínPicaridina (Bayrepel)* (AutanActivo)* (piperidín-carboxílico, 1-metilpropilester) (8h) (menor toxicidad); Dimetilftalato, aceites esenciales de plantas.

- Insecticidas residuales: Impregnar ropas con Permetrinas y mosquiteras tratadas con insecticidas ${ }^{5}$.

\section{Prevención secundaria}

Se basa en la quimioprofilaxis antipalúdica o toma de fármacos antipalúdicos a dosis preventivas antes, durante y después de la estancia en una zona palúdica. El tipo de medicación varía según la zona a visitar y dado que los antipalúdicos no están exentos de toxicidad, hay que hacer una valoración individualizada del riesgo. de forma absoluta. Para que la quimioprofilaxis tenga efectividad deberá continuarse durante las cuatro semanas siguientes al abandono de la zona palúdica, tanto si los viajeros vuelven a su país de origen como si van a una zona tropical exenta de paludismo, ya que el parásito que produce la enfermedad puede permanecer acantonado en el hígado durante ese período de tiempo. Asimismo cada vez son mayores las cepas de Plasmodium resistentes a los fármacos utilizados en la prevención y tratamiento de la enfermedad.

A pesar de todas estas recomendaciones, de todos los casos de paludismo importados, más del 75\% aparece en viajeros que no tomaron quimioprofilaxis, y el porcentaje se eleva al $85-97 \%$, si se suman los que tomaron la quimioprofilaxis de forma irregular. Datos muy importantes que coinciden con los obtenidos en este trabajo. En la Tabla 2, se recogen los principales fármacos utilizados en la prevención del paludismo.

Dentro de los países incluidos en los considerados como áreas endémicas de Paludismo se encuentra Afganistán.

La OMS, en su actualización de 2010, indica que existe riesgo de Paludismo desde mayo hasta noviembre, por debajo de 2.000 metros de altitud, lo que significa que 12 millones de afganos viven en áreas de riesgo, así como las tropas desplegadas 
Tabla 2. Principales fármacos utilizados en la prevención del Paludismo9

\begin{tabular}{|c|c|c|c|c|}
\hline Farmaco & Dosis & Indicaciones & Efectos adversos & $\begin{array}{l}\text { Contraindicaciones } \\
\text { Precauciones }\end{array}$ \\
\hline $\begin{array}{l}\text { Cloroquina. Resochin }{ }^{\circledR} \text {. } \\
\text { Comprimidos de } 150 \mathrm{mg} \text {. }\end{array}$ & $\begin{array}{l}2 \text { comprimidos una } \\
\text { vez a la semana. } \\
\text { Comezar una } \\
\text { semana antes de } \\
\text { empezar el viaje y } \\
\text { continuar un mes } \\
\text { después del regreso. }\end{array}$ & $\begin{array}{l}\text { Zonas donde no hay } \\
\text { resistencia del Plasmodio } \\
\text { a la cloroquina. }\end{array}$ & $\begin{array}{l}\text { Reacciones leves: dolor } \\
\text { abdominal, naúseas, } \\
\text { vómitos, diarrea. } \\
\text { Más raro: psicosis o } \\
\text { convulsiones. } \\
\text { Retinopatía: rara } \\
\text { cuando se usa como } \\
\text { profilaxis. }\end{array}$ & $\begin{array}{c}\text { Hipersensibilidad a } \\
\text { Cloroquina, deficit } \\
\text { G6PD*, retinopatía, } \\
\text { enfermedad SNC, } \\
\text { miastenia gravis, epilepsia, } \\
\text { psicosis, enfermedades } \\
\text { hematológicas. } \\
\text { Puede exacerbar los } \\
\text { síntomas de la psoriasis. }\end{array}$ \\
\hline $\begin{array}{l}\text { Mefloquina. Lariam }{ }^{\circledR} \text {. } \\
\text { Comprimidos de } 250 \mathrm{mg} \text {. }\end{array}$ & $\begin{array}{l}1 \text { comprimido a la } \\
\text { semana**. } \\
\text { Comenzar una } \\
\text { semana antes del } \\
\text { viaje y continuar } \\
\text { un mes despues } \\
\text { del regreso. }\end{array}$ & $\begin{array}{l}\text { Zonas de resistencia a } \\
\text { Cloroquina. Hay zonas } \\
\text { en el sudeste asiático } \\
\text { en las que hay descrita } \\
\text { resistencia de } \\
\text { P. falciparum a } \\
\text { Mefloquina. }\end{array}$ & $\begin{array}{c}\text { Neurológicos: SNC } \\
\text { y SNP. } \\
\text { Psiquiátricos: } \\
\text { alteraciones del } \\
\text { sueño, ansiedad, } \\
\text { trastornos del ánimo. } \\
\text { Las reacciones } \\
\text { neuropsiquiátricas } \\
\text { graves son raras } \\
\text { (1/10.000- } 1 / 13.000) . \\
\text { Síntomas } \\
\text { gastrointestinales. }\end{array}$ & $\begin{array}{c}\text { Contraindicado en } \\
\text { pacientes psiquiátricos, } \\
\text { epilepsia y arritmias } \\
\text { ventriculaes. } \\
\text { Precaución en personas } \\
\text { en las que se requiere } \\
\text { gran destreza (como los } \\
\text { pilotos). }\end{array}$ \\
\hline $\begin{array}{l}\text { Doxiciclina. } \\
\text { Comprimidos de } 100 \mathrm{mg} \text {. }\end{array}$ & $\begin{array}{l}1 \text { comprimido al } \\
\text { día. Comenzar un } \\
\text { día antes de entrar } \\
\text { en zona de riesgo y } \\
\text { continuar un mes } \\
\text { después del regreso. }\end{array}$ & $\begin{array}{l}\text { Zonas de resistencia } \\
\text { a Cloroquina y en } \\
\text { zonas de } P \text {. falciparum } \\
\text { multirresistente (no } \\
\text { se han encontrado } \\
\text { resistencias a } \\
\text { Doxiciclina). }\end{array}$ & $\begin{array}{c}\text { Fotosensibilidad } \\
(4-16 \%) . \\
\text { Trastornos } \\
\text { gastrointestinales: } \\
\text { naúseas, dolor } \\
\text { abdominal y esofagitis. } \\
\text { Candidiasis vaginal. }\end{array}$ & $\begin{array}{l}\text { Contraindicada en niños } \\
\text { menores de } 8 \text { años y } \\
\text { embarazadas. Precaución } \\
\text { en insuficiencia hepática. }\end{array}$ \\
\hline $\begin{array}{l}\text { Atovacuona+Proguanil. } \\
\text { Malarone }{ }^{\circledR} \text {. } \\
\text { Para adultos comprimidos } \\
\text { de } 100 \mathrm{mg} \text { de proguan } \\
\text { il/250 mg de atovacuona. } \\
\text { Pedátricos: } 25 \mathrm{mg} \text { de } \\
\text { proguanil } / 62.5 \mathrm{mg} \text { de } \\
\text { atovacuona. }\end{array}$ & $\begin{array}{l}1 \text { comprimido de } \\
\text { adulto al día para } \\
\text { viajeros de más } \\
\text { de } 40 \mathrm{~kg} \text {. } \\
\text { Comenzar } 1-2 \text { días } \\
\text { antes del viaje, } \\
\text { tomar diariamete } \\
\text { y continuar una } \\
\text { semana tras el } \\
\text { regreso. }\end{array}$ & $\begin{array}{l}\text { Zonas de resistencia } \\
\text { a Cloroquina y en } \\
\text { zonas de } P \text {. falciparum } \\
\text { multirresistente. }\end{array}$ & $\begin{array}{l}\text { Poco frecuentes dolor } \\
\text { abdominal, vómitos, } \\
\text { cefalea, insomnio, } \\
\text { fiebre y rash cutáneo. }\end{array}$ & $\begin{array}{l}\text { Contraindicado en } \\
\text { niños menores de } 5 \mathrm{~kg} \text {, } \\
\text { embarazadas, durante la } \\
\text { lactancia y en insuficiencia } \\
\text { renal severa (Aclaramiento } \\
\text { de Creatinina }<30 \text { ). }\end{array}$ \\
\hline
\end{tabular}

* Glucosa 6-Fosfato Deshidrogenasa

**Las mujeres, sobre todo las que tienen índice de masa corporal $<20 \mathrm{Kg} / \mathrm{m}$, tienen mayor propensión a sufrir efectos adversos. En estos casos se puede disminuir algo la dosis (3/4 de comprimido).

Fuente: López-Velez R. de Guzman M. cuadernos de malaria importada. Prevención en viajeros 2007.

en estas zonas (la ciudad de Herat se encuentra a 964 metros y Kabul a 1795 metros).

En el año 2003 (últimos datos disponibles según la OMS), hubo 591.441 casos confirmados de la enfermedad con una incidencia anual de 197/10.000 habitantes. El P. vivax representa el 93\% de todos los casos confirmados de malaria y $P$. falciparum explica el $7 \%$ restante, encontrándose entre el $0.002 \%$ en la provincia de Wardak hasta el $31 \%$ en la provincia de Takhar.
Se han notificado casos de Malaria en todos los ejércitos OTAN desplegados en la zona, 15 casos británicos durante el período comprendido entre 2001/02), 7 españoles entre 2002/04, 38 estadounidenses entre junio y septiembre del 2002 y 91 casos en 2011, y de la antigua URSS 7.683 casos entre 1981/89, también fueron notificados en diciembre de 2008, en el Role 2 francés en Camp Warehouse 21 casos de paludismo, 19 de ellos por P. falciparum y 2 por P. vivax ${ }^{10}$. 
Tabla 3. Zonas de riesgo de Paludismo y tipo de quimioprofilaxis recomendada ${ }^{10}$.

\begin{tabular}{|c|c|}
\hline Riesgo de paludismo & Tipo de profilaxis \\
\hline \multicolumn{2}{|l|}{ TIPO I } \\
\hline Riesgo muy limitado de transmisión de paludismo. & Sólo prevención de picadura de mosquito. \\
\hline \multicolumn{2}{|l|}{ TIPO II } \\
\hline $\begin{array}{l}\text { Riesgo de paludismo sólo por } P \text {. vivax o } P \text {. falciparum sensible } \\
\text { a Cloroquina. }\end{array}$ & Prevención de picadura de mosquito y profilaxis con Cloroquina. \\
\hline \multicolumn{2}{|l|}{ TIPO III } \\
\hline Riesgo de transmisión de paludismo por $P$. vivax o $P$. & Prevención de picadura de mosquito y profilaxis con \\
\hline falciparum, con emergencia de resistencia a Cloroquina. & Cloroquina-Proguanil. \\
\hline \multicolumn{2}{|l|}{ TIPO IV } \\
\hline $\begin{array}{l}\text { Riesgo alto de paludismo por } P \text {. falciparum, combinado con } \\
\text { evidencia de resistencia a antipalúdicos ó riesgo moderado/ } \\
\text { bajo de paludismo por } P \text {. falciparum en combinación con alto }\end{array}$ & $\begin{array}{l}\text { Prevención de picadura de mosquito y profilaxis con Mefloquina } \\
\text { o Doxiciclina o Atovacuona-Proguanil (según las resistencias de } \\
\text { cada zona). }\end{array}$ \\
\hline
\end{tabular}

grado de resistencia a fármacos.

Se ha evidenciado resistencia del P. falciparum a la cloroquina y la sulfadoxina-pirimetamina, por lo que la prevención recomendada es la tipo IV, dentro de los cuatro grupos que se refieren al tipo de prevención, expuestos en la Tabla 3. En resumen, mefloquina $\left(\right.$ Lariam $\left.{ }^{\circledR}\right)$ o atovacuona + proguanil $\left(\right.$ Malarone ${ }^{\circledR}$ ) o doxiciclina (Vibracina ${ }^{\circledR}$, Doxiclat ${ }^{\circledR}$ ), en función de las características de la misión. En el caso de los pilotos, personal de aeronaves o aquellos que manejen instrumentos que requieran alto poder de concentración y/o precisión, en general está contraindicada la mefloquina $\left(\operatorname{Lariam}^{\circledR}\right)$, pudiendo tomar atovacuona + proguanil o doxiciclina.

En cuanto a otras recomendaciones de Sanidad Exterior en Afganistán se exige certificado de vacunación contra la Fiebre Amarilla a los viajeros procedentes de países con riesgo de transmisión de la enfermedad. España no está incluida en estas zonas. En cuanto a las vacunas recomendadas es conveniente tener actualizado el calendario oficial de vacunaciones. Pueden ser recomendables otras vacunas, cuya prescripción deberá realizarse de forma personalizada en cualquiera de los Centros de Vacunación Internacional autorizados.

Durante el año 2013 se llevó a cabo el repliegue de la zona de Badghis, que comenzó el 21 de febrero, al entregar al Ejército afgano el puesto avanzado en Ludina. Posteriormente, se realizó la misma operación con los puestos avanzados de Moqur y Sanga Tesh, completándose el 25 de septiembre de 2013 con la entrega de la base de Qala-i-Naw, la principal base española en la provincia. Con ello culminó el repliegue español y la transferencia de todas las actividades de seguridad a las fuerzas afganas en esta provincia.

ASPFOR XXXIII ha sido la última rotación del contingente desplegado en la base "Ruy Gonzalez de Clavijo" en Qala-iNaw, la peculiaridad del repliegue y las características logísticas de esta misión también han influido en las cuestiones sanitarias en general, cada vez menos medios tanto humanos, al reducirse el número de células de estabilización, como materiales, y en la quimioprofilaxis en particular, como puede desprenderse del siguiente informe.
En la fase previa al despliegue ZO se lleva a cabo una Fase de Concentración a la que deben acudir todos los militares que van a ser desplegados en $\mathrm{ZO}$, en esta fase previa además de tener en cuenta aspectos operacionales y tácticos, todos los miembros de la misión deben obtener la máxima información sanitaria ya que se trata una medida básica para su propia seguridad. Esta información debe incluir todo tiempo de riesgos sanitarios a los que van estar expuestos los militares durante el despliegue $y$, fundamentalmente, las medidas para su prevención.

\section{MATERIAL Y MÉTODO}

Nuestro propósito ha sido llevar a cabo un estudio observacional transversal basándonos en los datos extraídos de los cuestionarios de salud que deben rellenar todos los militares antes de salir de Zona de Operaciones (ZO), una vez finalizada una misión internacional.

Las variables tenidas en cuenta son el grado de cumplimiento de la quimioprofilaxis total del grupo, y posteriormente distribuyendo a los sujetos en varios grupos en función de determinadas variables que son categorías profesionales, sexo y grupos de edad.

En los cuestionarios existe una primera parte a completar por el interesado y unas cuestiones finales que consisten en una serie de preguntas que efectúa el oficial médico.

En la parte a completar por el interesado las preguntas 9 y 10 son sobre el contacto con insectos y la prevención primaria y la 19 y la 20 son las que se refieren al tema de la quimioprofilaxis. En la 19 se interroga acerca de si se le recomendó realizar quimioprofilaxis y, en caso afirmativo, cual. En la 20 si la realizó adecuadamente y en caso de respuesta negativa, los motivos.

El primer dato a tener en cuenta se trata de la formulación de estas cuestiones ya que en el $79 \%$ de los casos el problema era el desconocimiento del significado del término quimioprofilaxis, ya que se trata de un término científico que hubo que 


\section{Cumplimiento de la quimioprofilaxis antipalúdica en ASPFOR XXXIII}

explicar uno por uno a casi el $80 \%$ de los interesados, y, a la vista de los resultados en esta pregunta, se trata de un término no apto para su uso en la población general. Además al responder a esta pregunta solían hacerlo de forma negativa pero al preguntar en cada caso, la mayoría se refería a que no la había llevado a cabo, no a que no se lo hubieran recomendado en la fase previa al despliegue en $\mathrm{ZO}$.

En cuanto a los motivos más frecuentes para no iniciar la quimioprofilaxis se encuentran por orden de frecuencia: motivos personales, por decisión propia, por miedo a efectos adversos, lo que en resumen se basa en una mala percepción del riesgo beneficio. $\mathrm{Y}$ en el caso de abandono del tratamiento lo más frecuente es que se deba, en primer lugar, al hecho de no haber visto mosquitos en zona o por efectos adversos. De estos, los más frecuentemente referidos, son el mareo, las molestias gastrointestinales y la cefalea. También cabe destacar en este último grupo aquellos que no han iniciado la quimioprofilaxis por haber presentado alguno de estos efectos adversos al tomarla en misiones previas. No hubo ninguna referencia expresa a efectos adversos psiquiátricos.

Otro aspecto muy importante es la incorrecta realización de la quimioprofilaxis. En este caso también influye la gestión de la medicación. Hay unidades en las que se entrega la medicación de forma semanal al interesado, que frecuentemente no puede recogerla por cuestiones logísticas, derivadas de las operaciones, o por olvido. En otras unidades, se le entregan las cajas de una en una y en otras acuden al servicio de sanidad para recoger la medicación, lo que también repercute en el cumplimiento. También son muy frecuentes los olvidos derivados de la pauta de administración semanal, en el contexto de una rutina en la que "todos los días son iguales" y es difícil acordarse cuando uno está centrado en cuestiones mucho más relevantes. Cuando estos olvidos se repiten también hemos recogido casos que deciden abandonarlo por lo errático del cumplimiento y por percibir que de esta forma es ineficaz.

\section{RESULTADOS}

Para obtenerlos, como ya se ha mencionado, hemos tomado los datos de los cuestionarios de salud y las entrevistas llevadas a cabo por el Oficial Médico justo antes del regreso a territorio nacional. Se ha dividido a los participantes según el empleo militar, sexo y en cuatro grupos de edad para su clasificación.

Teniendo en cuenta todas estas cuestiones y basándonos en los cuestionarios de salud realizados por los oficiales del Cuerpo Militar de Sanidad (CMS) desplegados en ASPFOR XXXIII en la base "Ruy Gonzalez de Clavijo" en Qala-i-Naw, hasta un $78 \%$ del personal no llevó a cabo la quimioprofilaxis antipalúdica de forma adecuada. Un $61 \%$ ni siquiera inició la quimioprofilaxis por pensar que era mayor el riesgo que el beneficio, y hubo un $17 \%$ de abandonos o incumplimientos, justificándose sólo el $22 \%$ de éstos por efectos adversos atribuibles a la medicación.

Por categorías profesionales, la realización correcta de la quimioprofilaxis alcanzó su cifra más elevada entre los oficiales, casi un $42 \%$, en los suboficiales el $25 \%$ y en personal de tropa tan solo el $11 \%$.
Por géneros el cumplimiento en las mujeres fue significativamente mayor, siendo de un $38 \%$ frente al $22 \%$ de los hombres.

Por edades se dividió a la población de forma aleatoria en 4 grupos, menores de 25 años, entre 25 y 35 años, entre 35 y 45, y mayores de 45. El incumplimiento, aún siendo elevado en todos los grupos, fue algo mayor en los dos primeros, como reflejan las siguientes cifras: primer grupo ( $<25$ años) $86 \%$, segundo grupo (25-35 años) $82 \%$, tercer grupo (35-45años) $70 \%$ y cuarto grupo (>45 años) $74 \%$.

Asimismo son básicos los errores en cuanto al inicio y fin de la quimioprofilaxis. Un ejemplo significativo en esta rotación ha sido el hecho de que un $37 \%$ de los que estaban realizando de manera adecuada la quimioprofilaxis dejaron de hacerlo cuando su unidad se replegó hacia la base de Herat por dejar de percibir el riesgo de enfermedad en esta última ya que no se les había informado acerca de esta cuestión en concreto. Además hasta un 57\% desconocían que debían continuar con la quimioprofilaxis durante 4 semanas tras su llegada a territorio nacional. Y reinterrogando a los interesados, hasta en un $40 \%$ de los casos, el inicio de la medicación previa al despliegue tampoco se había realizado correctamente, empezando a tomar la medicación a la llegada a ZO.

\section{DISCUSIÓN}

A pesar de la dilatada experiencia de los ejércitos de múltiples nacionalidades en misiones internacionales con despliegue en áreas endémicas para el paludismo, no existen publicaciones fiables en cuanto a la adherencia a la quimioprofilaxis en ZO. Lo que si hemos podido encontrar en la búsqueda bibliográfica realizada, son descripciones de casos de paludismo en casi todos los ejércitos que han participado en estas operaciones.

Por esto no parece que, hasta el momento, se puedan establecer datos comparables con los de nuestro estudio. Que por otra parte, y como ya se ha mencionado, tan solo se trata de unos datos, a partir de una muestra muy concreta, obtenidos en la última rotación de ASPFOR y con la peculiaridad de tratarse de la encargada del repliegue.

\section{CONCLUSIONES}

Con los datos obtenidos en esta rotación de ASPFOR XXXIII, podemos decir que no se han conseguido unos objetivos adecuados en cuanto al cumplimiento de la profilaxis antipalúdica. A pesar de los esfuerzos que se realizan en cuanto a formación sanitaria, estos han sido insuficientes en este caso concreto.

Si estos datos se extrapolaran al cumplimiento de las recomendaciones de quimioprofilaxis en otras rotaciones y a otras misiones internacionales, parece claro que hay que plantearse nuevas técnicas de concienciación del riesgo de contraer paludismo en zonas endémicas, así como la incidencia real y gravedad de los posibles efectos secundarios para poder conocer el balance riesgo-beneficio, además sería importante implantar medidas destinadas a controlar el cumplimiento terapéutico. 


\section{Arcos Sánchez y FT. Salinas Vela}

\section{BIBLIOGRAFÍA}

1. World Health Organization International travel a health. Actualización en 2014. Disponible en: http://www.who.int/ith/diseases/malaria/en

2. Ministerio de Sanidad y Consumo. Viajes internacionales y salud [página en Internet]. Ministerio de Sanidad y Consumo. Actualización 2014. Disponible en: https://www.msssi.gob.es/profesionales/saludPublica/sanidadExterior/salud/viajesInter/cap7.htm

3. Guía de Enfermedades infecciosas importadas. Madrid: Ministerio de Sanidad y Consumo; 2008 (consulta el 25 de enero de 2014). Disponible en: http:// www.msssi.gob.es/profesionales/saludPublica/prevPromocion/promocion/ migracion/docs/GuiaEnfInfImp.pdf

4. Fernández-Sierra MA, Rosales Rodríguez M. Atención sanitaria al viajero internacional. Med Fam Andal 2009; 10 (3): 234-41.

5. Informe Mundial sobre el Paludismo 2013 World Health Organization Disponible en: http://www.who.int/malaria/publications/world_malaria_report_2013/report/en

6. Quimioterapia práctica del paludismo. Informe de un Grupo Científico de la OMS, 1990. Serie de Informes Técnicos, N 805). Disponible en: http://apps. who.int/iris/handle/10665/40290?locale $=$ ar
7. Guidelines for the treatment of malaria, 2nd edition. World Health Organization, 2010 (consulta 25 de enero de 2014). Disponible en: http://www.who. int/malaria/publications/atoz/9789241547925/en

8. Aplicación de la resolución 66/289 de la Asamblea General sobre la consolidación de los logros y aceleración de la labor destinada a combatir y erradicar la malaria en los países en desarrollo, particularmente en los de África, para 2015. (consulta el 25 de enero de 2014). Disponible en: http:// www.who.int/malaria/publications/atoz/UNGA_malaria_report_2013 Spanish.pdf

9. Boletín Epidemiológico de las Fuerzas Armadas. VOL. 19 N$^{\circ} 230$ Diciembre 2012.

10. Boletín Epidemiológico de las Fuerzas Armadas. VOL. $17 \mathrm{~N}^{\circ} 202$ Agosto 2010 .

11. Ministerio de Sanidad y Consumo. Viajes internacionales y salud. Ministerio de Sanidad y Consumo (actualización en 2014). Disponible en: https://www. msssi.gob.es/profesionales/saludPublica/sanidadExterior/salud/viajesInter/ cap7.htm.

12. World Health Organization. World Malaria Report 2013. Disponible en: http://www.who.int/malaria/publications/world_malaria_report_2013/report/en]. 